\title{
Exploring vector-borne infection ecology in multi-host communities: a case study of West Nile virus
}

\author{
Giovanni Marini ${ }^{\mathrm{a}, \mathrm{b}, *}$, Roberto Rosáa ${ }^{\text {, Andrea } \text { Pugliese }^{\mathrm{b}} \text {, Hans Heesterbeek }}{ }^{\mathrm{c}}$ \\ ${ }^{a}$ Department of Biodiversity and Molecular Ecology, Research and Innovation Centre, \\ Fondazione Edmund Mach, Via E. Mach 1, 38010 San Michele all'Adige (TN), Italy \\ ${ }^{b}$ Department of Mathematics, University of Trento, via Sommarive 14, 38123 Povo \\ (Trento), Italy \\ ${ }^{c}$ Faculty of Veterinary Medicine, University of Utrecht, Yalelaan 7, 3584 CL Utrecht, The \\ Netherlands
}

\begin{abstract}
In this study, we develop a model to investigate how ecological factors might affect the dynamics of a vector-borne pathogen in a population composed by different hosts which interact with each other. Specifically, we consider the case when different host species compete with each other, as they share the same habitat, and the vector might have different feeding preference, which can also be time dependent. As a prototypical example, we apply our model to study the invasion and spread, during a typical season, of West Nile virus in an ecosystem composed of two competent avian host species and possibly of deadend host species. We found that competition and vector feeding preferences can profoundly influence pathogen invasion, influencing its probability to start an epidemic, and influencing transmission rates. Finally, when considering timedependent feeding preferences, as observed in the field, we noted that the virus circulation could be amplified and that the timing of epidemic peaks could be changed. Our work highlights that ecological interactions between hosts can have a profound influence on the dynamics of the pathogen and that, when modeling vector-borne infections, vector feeding behavior should, for this reason, be carefully evaluated.
\end{abstract}

\footnotetext{
* Corresponding author

Email address: giovanni.marini@fmach.it (Giovanni Marini)
} 
Keywords: Epidemiology, mosquito-borne infection, competition, vector feeding preference.

1

3

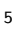

\section{Introduction}

Ecological interactions within and between species, such as competition and consumer-resource relations, can be influenced by infection dynamics of pathogens and parasites, and vice versa. A recent attempt to more systematically address their mutual interactions (Roberts and Heesterbeek, 2013) has focused on invasion of infection into ecological communities. For this purpose, they computed the basic reproduction number $\mathcal{R}_{0}$, defined as the average number of new cases of an infection caused by one typical infected individual, in a population consisting of susceptibles only (Diekmann et al., 2010).

Following Roberts and Heesterbeek (2013), we investigate the case of a vectorborne infection spreading in a population where different species of hosts compete with each other, for instance for food or habitat, and where the vector bites the hosts according to its feeding preferences. Several papers investigating an infection spreading into competing hosts have been published (Bowers and Turner, 1997; Han and Pugliese, 2009; Bokil and Manore, 2013), but to the best of our knowledge none analyzing the case of a vector-borne infection. In this type of infection, the pathogen is usually transmitted to and from the host when the latter is bitten by the vector to obtain a blood meal required for reproduction.

We focus on mosquito-borne infections and analyze a basic system where one vector species takes blood meals from two competent host species that compete ecologically. We show how to compute $\mathcal{R}_{0}$ allowing for different reservoir host competence (i.e. probability of transmitting the pathogen to the vector). As a prototypical example, we consider West Nile virus (WNV) in an ecosystem composed of two avian host species. However, the formula for $\mathcal{R}_{0}$ is easily generalizable to ecosystems of arbitrary numbers of host and non-host species that interact by competition and/or food web relations (Roberts and Heesterbeek, 
2013).

WNV is a flavivirus first isolated in Uganda in 1937 (Smithburn et al., 1940) and now present on every continent (Reisen, 2013). It is maintained in a birdmosquito transmission cycle primarily involving mosquitoes belonging to Culex species, of which the pipiens sub-complex is thought to be one of the most important in Europe (Zeller and Schuffenecker, 2004) and North America (Reisen, 2013). Humans and other mammals (e.g. horses) are considered dead-end hosts, i.e. they can not transmit the virus.

Culex mosquitoes and WNV have a broad host range, and mosquito feeding preferences can change during the season. In fact mosquitoes seem to preferentially bite certain hosts more than others, even if they are less available (Kilpatrick et al., 2006a; Thiemann et al., 2011; Simpson et al., 2012; Taylor et al., 2012; Rizzoli et al., 2015); moreover its preferences seem to change during the breeding season (Kilpatrick et al., 2006b; Thiemann et al., 2011; Burkett-Cadena et al., 2012).

Many models have been proposed to study West Nile virus dynamics among different bird species (Cruz-Pacheco et al., 2005, 2012; Maidana and Yang, 2011; Simpson et al., 2012) but they do not explicitly investigate ecological interactions between the hosts or the effects of changes in host preference over the season. Our aim is to investigate how ecological interactions, such as competition, and shifting mosquito feeding preferences can affect the invasion of a pathogen and therefore change the outcome relatively to a baseline scenario which does not include such features.

\section{The model}

We analyze the simplest case with only two competing species, both competent hosts for an infection transmitted by a vector with population size $V$. In addition, we assume that hosts can not recover, but may die due to the infection. To this aim, we develop a compartmental model similar to the one proposed by Lord et al. (1996) with hosts and vectors classified according to whether they 
$$
\left\{\begin{aligned}
N_{1}^{\prime} & =r_{1}\left(1-\frac{N_{1}+c_{12} N_{2}}{K_{1}}\right) N_{1}-\alpha_{1} I_{1} \\
N_{2}^{\prime} & =r_{2}\left(1-\frac{N_{2}+c_{21} N_{1}}{K_{2}}\right) N_{2}-\alpha_{2} I_{2} \\
V^{\prime} & =\left(n_{E} \sigma b_{\max }\left(1-\rho_{V} V\right)-\mu_{V}\right) V \\
I_{1}^{\prime} & =\left[p_{V 1} b_{1} \frac{I_{V}}{N_{1}}+\beta_{11} p_{11} \frac{I_{1}}{N_{1}}+\beta_{12} p_{21} \frac{I_{2}}{N_{2}}\right] S_{1}-\left(\alpha_{1}+\mu_{1}+r_{1} \frac{a_{11} N_{1}+a_{12} c_{12} N_{2}}{K_{1}}\right) I_{1} \\
I_{2}^{\prime} & =\left[p_{V 2} b_{2} \frac{I_{V}}{N_{2}}+\beta_{22} p_{22} \frac{I_{2}}{N_{2}}+\beta_{21} p_{12} \frac{I_{1}}{N_{1}}\right] S_{2}-\left(\alpha_{2}+\mu_{2}+r_{2} \frac{a_{22} N_{2}+a_{21} c_{21} N_{1}}{K_{2}}\right) I_{2} \\
I_{V}^{\prime} & =\left[p_{1 V} b_{1} \frac{I_{1}}{N_{1}}+p_{2 V} b_{2} \frac{I_{2}}{N_{2}}\right] S_{V}+\left(q_{V} n_{E} \sigma b_{\max }\left(1-\rho_{V} V\right)-\mu_{V}\right) I_{V}
\end{aligned}\right.
$$

are susceptible $S$ or infected $I$. Although vector-borne infections are usually transmitted only by the vector, we consider also the possibility of host-to-host transmission, as this has been shown to be possible for West Nile virus among crows by Komar et al. (2003).

To model the competition among birds we assume, as in (Gamarra et al., 2005), that they both follow a Lotka-Volterra dynamics. In addition, the mosquito population dynamics is assumed to be density dependent; in particular, we assume that density can affect larval development and survival, as observed by Agnew et al. (2010).

The equations of the model are

where

- $N_{i}=S_{i}+I_{i}$ is the number of individuals of species $i$ with $i \in\{1,2\}$;

- $r_{i}=\eta_{i}-\mu_{i}>0$ is the growth rate of species $i \in\{1,2\}$, where $\eta_{i}$ and $\mu_{i}$ are the birth and death rate respectively. They are assumed not to be influenced by the vector. Each species has a certain carrying capacity $K_{i}$;

- $c_{i j}$ represents the effect of competition of species $j$ on species $i$ with $i, j \in\{1,2\}, i \neq j$;

- $a_{i j} \in[0,1]$ is the proportion of competition from species $j$ that affects the death rate of species $i$ with $i, j \in\{1,2\}$;

- $n_{E}$ is the number of eggs laid by a gravid mosquito and $\sigma$ is the probability that an egg becomes an adult; 
- $b_{\max }$ is the vector biting rate, which can be thought of as the inverse of the length of the gonotrophic cycle, i.e. the interval spanned between the blood meal and the oviposition. Bites are divided between the two host populations with $b_{1}, b_{2}$, denoting the biting rates on species 1 and 2 respectively, and therefore $b_{\max }=b_{1}+b_{2}$;

- $\rho_{V}<1$ is the density dependent factor on vector fecundity. We can then define $K_{V}$ as the vector carrying capacity, as follows:

$$
K_{V}:=\frac{1}{\rho_{V}}\left(1-\frac{\mu_{V}}{n_{E} \sigma b_{\max }}\right) .
$$

- $\mu_{V}$ is the vector death rate;

- $\alpha_{i}$ is the additional death rate for species $i$ due to the infection;

- $p_{i j}$ is the probability that an infected individual of type $i \in\{1,2, V\}$ infects a susceptible individual of type $j \in\{1,2, V\}$, given contact or bite;

- $\beta_{i j}$ is the direct transmission rate between host species $i$ and $j$;

- $q_{V}$ is the probability of vertical transmission, i.e. the probability that an infected mosquito passes the virus to its offspring.

If there is no infection, the Jacobian of system (1) at the Infection-free Equilibrium is given by

$$
J=\left(\begin{array}{ll}
C & D \\
0 & H
\end{array}\right)
$$

where

$$
C=\left(\begin{array}{ccc}
\frac{r_{1}}{K_{1}}\left(K_{1}-c_{12} N_{2}-2 N_{1}\right) & -\frac{c_{12} r_{1} N_{1}}{K_{1}} & 0 \\
-\frac{c_{21} r_{2} N_{2}}{K_{2}} & \frac{r_{2}}{K_{2}}\left(K_{2}-c_{21} N_{1}-2 N_{2}\right) & 0 \\
0 & 0 & n_{E} \sigma b_{\max }\left(1-2 \rho_{V} V\right)-\mu_{V}
\end{array}\right)
$$


dynamics of the two host species and the vector species:

$$
H=\left(\begin{array}{ccc}
p_{11} \beta_{11}-\left(\alpha_{1}+\tilde{\mu}_{1}\right) & \frac{\beta_{12} p_{21} N_{1}}{N_{2}} & p_{V 1} b_{1} \\
\frac{p_{12} \beta_{21} N_{2}}{N_{1}} & \beta_{22} p_{22}-\left(\alpha_{2}+\tilde{\mu}_{2}\right) & p_{V 2} b_{2} \\
\frac{p_{1 V} b_{1} V}{N_{1}} & \frac{p_{2 V} b_{2} V}{N_{2}} & q_{V} n_{E} \sigma b_{\max }\left(1-\rho_{V} V\right)-\mu_{V}
\end{array}\right)
$$

${ }_{98} \quad$ where $\tilde{\mu}_{i}=\mu_{i}+r_{i} \frac{a_{i i} N_{i}+a_{i j} c_{i j} N_{j}}{K_{i}}$ with $i \in\{1,2\}$.

99 The matrix $D$ in the upper right corner is

$$
D=\left(\begin{array}{ccc}
-\alpha_{1} & 0 & 0 \\
0 & -\alpha_{2} & 0 \\
0 & 0 & 0
\end{array}\right)
$$

100

The infection-free non trivial equilibrium is

$$
N_{1}^{*}=\frac{K_{1}-c_{12} K_{2}}{1-c_{12} c_{21}}, \quad N_{2}^{*}=\frac{K_{2}-c_{21} K_{1}}{1-c_{12} c_{21}}, \quad V^{*}=K_{V}
$$

which exists (i.e. $N_{1}^{*} \geq 0, N_{2}^{*} \geq 0, V^{*} \geq 0$ ) and is stable provided

$$
K_{i}>c_{i j} K_{j}, \quad \mu_{V}<n_{E} \sigma b_{\max }
$$

102 We observe that $N_{i}^{*} / K_{i}>N_{j}^{*} / K_{j}$ (i.e. population size of species $i$ is depressed by competition less than species $j$ ) when $c_{j i} \frac{K_{i}}{K_{j}}>c_{i j} \frac{K_{j}}{K_{i}}$. In particular when $K_{i}=K_{j}$ (which can also be assumed through an appropriate scaling) we can equate size of competition coefficients with depression of population size.

106 As in (Roberts and Heesterbeek, 2013) we write $H=T+\Sigma$ where $T$ is the epidemiological transmission matrix

$$
T=\left(\begin{array}{ccc}
p_{11} \beta_{11} & \frac{\beta_{12} p_{21} N_{1}^{*}}{N_{2}^{*}} & p_{V 1} b_{1} \\
\frac{p_{12} \beta_{21} N_{2}^{*}}{N_{1}^{*}} & \beta_{22} p_{22} & p_{V 2} b_{2} \\
\frac{p_{1 V} b_{1} V^{*}}{N_{1}^{*}} & \frac{p_{2 V} b_{2} V^{*}}{N_{2}^{*}} & q_{V} n_{E} \sigma b_{\max }\left(1-\rho_{V} V^{*}\right)
\end{array}\right)
$$


and $\Sigma$ is the epidemiological transition matrix

$$
\Sigma=\left(\begin{array}{ccc}
-\left(\alpha_{1}+\tilde{\mu}_{1}\right) & 0 & 0 \\
0 & -\left(\alpha_{2}+\tilde{\mu}_{2}\right) & 0 \\
0 & 0 & -\mu_{V}
\end{array}\right)
$$

and therefore the so called next-generation matrix with large domain (Diekmann et al., 2010) is

$$
\mathcal{K}=-T \Sigma^{-1}=\left(\begin{array}{ccc}
\frac{p_{11} \beta_{11}}{\left(\alpha_{1}+\tilde{\mu}_{1}\right)} & \frac{\beta_{12} p_{21} N_{1}^{*}}{N_{2}^{*}\left(\alpha_{2}+\tilde{\mu}_{2}\right)} & \frac{p_{V 1} b_{1}}{\mu_{V}} \\
\frac{p_{12} \beta_{21} N_{2}^{*}}{N_{1}^{*}\left(\alpha_{1}+\tilde{\mu}_{1}\right)} & \frac{\beta_{22} p_{22}}{\left(\alpha_{2}+\tilde{\mu}_{2}\right)} & \frac{p_{V 2} b_{2}}{\mu_{V}} \\
\frac{p_{1 V} b_{1} V^{*}}{N_{1}^{*}\left(\alpha_{1}+\tilde{\mu}_{1}\right)} & \frac{p_{2 V} b_{2} V^{*}}{N_{2}^{*}\left(\alpha_{2}+\tilde{\mu}_{2}\right)} & q_{V}
\end{array}\right)
$$

and $\mathcal{R}_{0}$ is the dominant eigenvalue of $\mathcal{K}$.

\subsection{Infections without horizontal transmission}

This is probably the most common case, since, as explained above, most vector-borne infections are transmitted only by the vector, so $p_{i j}=0, i, j \in\{1,2\}$. In this case the next-generation matrix becomes

$$
\mathcal{K}=\left(\begin{array}{ccc}
0 & 0 & \frac{p_{V 1} b_{1}}{\mu_{V}} \\
0 & 0 & \frac{p_{V 2} b_{2}}{\mu_{V}} \\
\frac{p_{1 V} b_{1} V^{*}}{N_{1}^{*}\left(\alpha_{1}+\tilde{\mu}_{1}\right)} & \frac{p_{2 V} b_{2} V^{*}}{N_{2}^{*}\left(\alpha_{2}+\tilde{\mu}_{2}\right)} & q_{V}
\end{array}\right)
$$

and the formula for $\mathcal{R}_{0}$ is

$$
\mathcal{R}_{0}=\frac{1}{2}\left(\sqrt{\frac{4 p_{V 1} p_{1 V} b_{1}^{2}}{\mu_{V}\left(\alpha_{1}+\tilde{\mu}_{1}\right)} \frac{V^{*}}{N_{1}^{*}}+\frac{4 p_{V 2} p_{2 V} b_{2}^{2}}{\mu_{V}\left(\alpha_{2}+\tilde{\mu}_{2}\right)} \frac{V^{*}}{N_{2}^{*}}+q_{V}^{2}}+q_{V}\right)
$$

with $N_{1}^{*}, N_{2}^{*}, V^{*}$ as in (2).

We note that the biting rates $b_{1}, b_{2}$ play a crucial role for $\mathcal{R}_{0}$, which depends also on the vector to host ratio which is in turn driven by the competition coefficients and the carrying capacities.

In order to make (3) more perspicuous, we can simplify it by assuming that an 
infected vector passes the pathogen to any susceptible host with same probability $p_{V 1}=p_{V 2}=p_{V H}$ and that it can not transmit the virus to its offspring (so $\left.q_{V}=0\right)$. We can further assume that mosquitoes bite hosts according to their density, so $b_{i}=b_{\max } \frac{N_{i}}{N_{1}+N_{2}}$ with $i \in\{1,2\}$. In this case (3) reduces to

$$
\mathcal{R}_{0}=\frac{b_{\max }}{N_{1}^{*}+N_{2}^{*}} \sqrt{\frac{p_{V H} V^{*}}{\mu_{V}}} \sqrt{\frac{p_{1 V} N_{1}^{*}}{\alpha_{1}+\tilde{\mu}_{1}}+\frac{p_{2 V} N_{2}^{*}}{\alpha_{2}+\tilde{\mu}_{2}}}
$$

with $\mathbf{N}_{\mathbf{1}}^{*}, \mathbf{N}_{\mathbf{2}}^{*}$ as in (2). From (4) we see that competition does not affect $\mathcal{R}_{0}$ linearly. In fact, for fixed $c_{21}$, increasing $c_{12}$ will decrease $N_{1}^{*}$ while at the same time $N_{2}^{*}$ and $\tilde{\mu}_{1}$ will increase. Thus one term inside the square root will increase with $c_{12}$ while the other will decrease; as a consequence, the overall effect on $\mathcal{R}_{0}$ is not straightforward.

\subsection{Horizontal transmission}

In this case we assume, as observed for WNV (Komar et al., 2003), that horizontal transmission can happen only between individuals belonging to the same species, so $p_{i j}=0, i \neq j$. In this case

$$
\mathcal{K}=\left(\begin{array}{ccc}
\frac{p_{11} \beta_{11}}{\left(\alpha_{1}+\tilde{\mu}_{1}\right)} & 0 & \frac{p_{V 1} b_{1}}{\mu_{V}} \\
0 & \frac{p_{22} \beta_{22}}{\left(\alpha_{2}+\tilde{\mu}_{2}\right)} & \frac{p_{V 2} b_{2}}{\mu_{V}} \\
\frac{p_{1 V} b_{1} V^{*}}{N_{1}^{*}\left(\alpha_{1}+\tilde{\mu}_{1}\right)} & \frac{p_{2 V} b_{2} V^{*}}{N_{2}^{*}\left(\alpha_{2}+\tilde{\mu}_{2}\right)} & q_{V}
\end{array}\right) .
$$

$\mathcal{R}_{0}$ is then the largest root of a 3 -rd order equation. We will consider some numerical examples in the next section.

Finally, in order to investigate the combined effect of horizontal and vector transmission, we consider, for the sake of simplicity, that there is only one host species, say species 1 . Then, with only horizontal transmission

$$
\mathcal{R}_{0}^{h}=\frac{p_{11} \beta_{11}}{\left(\alpha_{1}+\tilde{\mu}_{1}\right)},
$$

while, with only vector transmission (and $\left.q_{V}=0\right)$,

$$
\mathcal{R}_{0}^{V}=\sqrt{\frac{p_{V 1} p_{1 V} b_{1}^{2} V^{*}}{\mu_{V} N_{1}^{*}\left(\alpha_{1}+\tilde{\mu}_{1}\right)}} .
$$


When both transmission routes operate, one obtains

$\mathcal{R}_{0}=\sqrt{\left(\frac{p_{11} \beta_{11}}{4\left(\alpha_{1}+\tilde{\mu}_{1}\right)}\right)^{2}+\frac{p_{V 1} p_{1 V} b_{1}^{2} V^{*}}{\mu_{V} N_{1}^{*}\left(\alpha_{1}+\tilde{\mu}_{1}\right)}}+\frac{p_{11} \beta_{11}}{\alpha_{1}+\tilde{\mu}_{1}}=\sqrt{\frac{\left(\mathcal{R}_{0}^{h}\right)^{2}}{4}+\left(\mathcal{R}_{0}^{V}\right)^{2}}+\frac{\mathcal{R}_{0}^{h}}{2}$.

From (5) we can note that $\mathcal{R}_{0} \leq(>) 1 \Longleftrightarrow \mathcal{R}_{0}^{h}+\left(\mathcal{R}_{0}^{V}\right)^{2} \leq(>) 1$

\section{Numerical example}

Here, we present a numerical example to explore the influence of vector and host ecology, in our setting, on invasion of the infectious agent. In particular, we study the invasion of WNV with two bird species. We selected their respective parameters among the most competent species, that are American crow (Corvus brachyrhynchos, species 1) and House finch (Haemorhous mexicanus, species 2), as found in (Komar et al., 2003). We assume horizontal transmission only in species 1 (American crow), since Komar et al. (2003) found its occurrence in this species only, and assume $p_{22}=0$.

Finally, we also assume that the vector has a fixed daily biting rate $b_{\max }=b_{1}+b_{2}$. The baseline parameters, with their description, are reported in Table 1.

\subsection{Effect of vector and host ecology on $\mathcal{R}_{0}$}

We assume that the vector bites its hosts according to their density with a fixed daily rate $b_{\max }=0.2$, and $a_{11}=a_{22}=a_{12}=a_{21}=0.5$. In Figure 1 the effect of competition on $\mathcal{R}_{0}$ is shown. In Figure 1a (left panel) we can see that for a fixed value of $c_{i j}$, increasing $c_{j i}$ will increase $\mathcal{R}_{0}$ and the highest values are reached when $c_{12}$ is particularly large, so when species 1 (which has a higher probability of transmitting the virus to the vector) is much less abundant than the other. The lowest values are expected when the competition is not very high. We remark that $\mathcal{R}_{0}$ is always greater than 1 and, as expected from formula (5), it is also greater than the one computed without vector transmission. In fact in this latter case $\mathcal{R}_{0}^{h} \sim 0.73$ (see Supplementary Material), thus mosquitoes are crucial for the pathogen invasion and transmission. 


\begin{tabular}{llcc} 
Parameter & Description & Value & Source \\
\hline$p_{V 1}, p_{V 2}$ & Transmission probability mosquito to bird & 0.88 & (Turell et al., 2001) \\
$p_{1 V}$ & Transmission probability species 1 to mosquito & 0.5 & (Komar et al., 2003) \\
$p_{2 V}$ & Transmission probability species 2 to mosquito & 0.28 & (Komar et al., 2003) \\
$p_{11} \beta_{11}$ & Contact transmission rate in crows & 0.33 & (Hartemink et al., 2007) \\
$\mu_{V}$ & Death rate in mosquitoes (/day) & 0.08 & (Hartemink et al., 2007) \\
$q_{V}$ & Transovarial transmission rate & 0.004 & (Hartemink et al., 2007) \\
$\alpha_{1}$ & Species 1 WNV-related mortality rate (/day) & 0.2 & (Komar et al., 2003) \\
$\alpha_{2}$ & Species 2 WNV-related mortality rate (/day) & 0.11 & (Komar et al., 2003) \\
$n_{E}$ & Number of mosquito eggs in one batch & 200 & (Hartemink et al., 2007) \\
$\sigma$ & Survival probability egg to female mosquito & 0.1 & (Hartemink et al., 2007) \\
$\mu_{1}, \mu_{2}$ & Bird death rate & 0.001 & (Bowman et al., 2005) \\
$r_{1}, r_{2}$ & Bird growth rate & 0.5 & (Bowman et al., 2005) \\
$\frac{K_{V}}{\left(K_{1}+K_{2}\right)}$ & Mosquito to bird ratio & 5 & (Cruz-Pacheco et al., 2005) \\
$K_{1}, K_{2}$ & Carrying capacities for birds & 1000 & Assumption \\
$a_{11}, a_{12}, a_{21}, a_{22}$ & Proportion of competition affecting the death rate & Varying &
\end{tabular}

Table 1: parameters.

Figure 1b (right panel) shows how $\mathcal{R}_{0}$ is influenced by competition and its contribution to the death rate, represented by $a_{12}, a_{21}$. $\mathcal{R}_{0}$ is greater when host death rates are less affected by competition (as this increases expected life of infected individuals) and, in all three cases, it increases linearly with $c_{12}=c_{21}$. Since the three cases $\left(a_{12}=a_{21}=0.1,0.5,0.9\right)$ do not differ substantially, from now on we consider only the case with $a_{12}=a_{21}=a_{22}=a_{11}=0.5$.

As observed in many field studies, Culex pipiens may show different feeding preferences for different avian species (Kilpatrick et al., 2006a; Simpson et al., 2012; Thiemann et al., 2011). We say that a species, say $i$, is preferred if $\frac{b_{i}}{b_{\max }}>\frac{N_{i}}{N_{i}+N_{j}}$, i.e. its fraction of bites is higher than its frequency. Hence we can model the mosquito biting preference introducing, as in (Simpson et al., 2012), the feeding preference index $\delta_{V}, \delta_{V} \geq 1$. According to this the biting rates become

$$
b_{i}=b_{\max } \frac{\delta_{V} N_{i}}{\delta_{V} N_{i}+N_{j}}, \quad b_{j}=b_{\max } \frac{N_{j}}{\delta_{V} N_{i}+N_{j}} .
$$

179 We study the cases $b_{\max }=0.1,0.2,0.3$; as the biting rate can be interpreted as the reciprocal of the duration of the gonotrophic cycle, we are assuming that it 

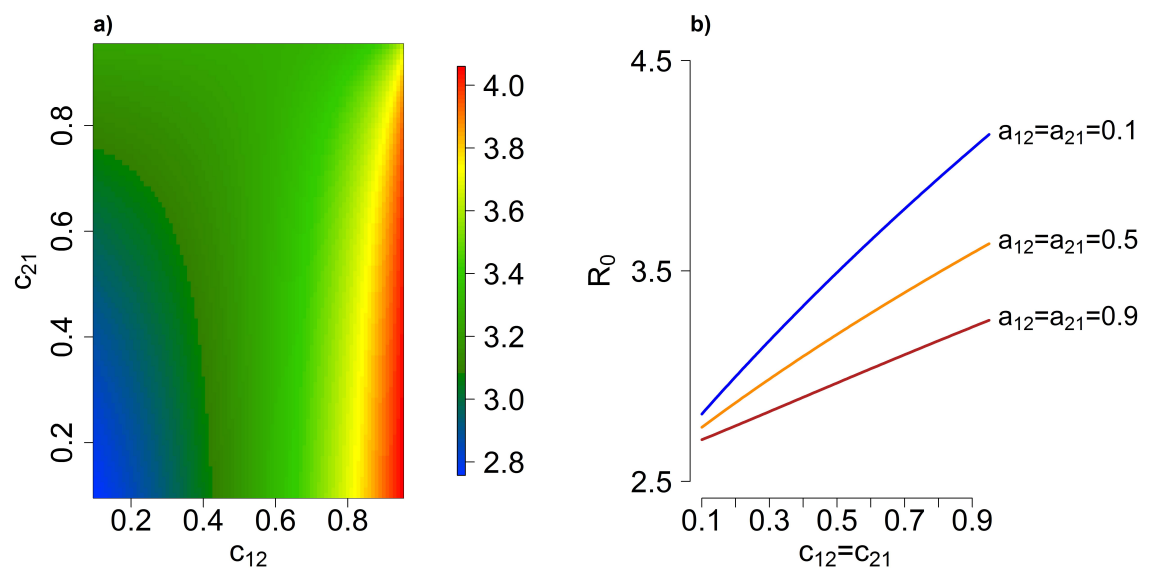

Figure 1: Panel a) $\mathcal{R}_{0}$ according to the competition coefficients with $a_{12}=a_{21}=0.5$. Panel b) $\mathcal{R}_{0}$ according to the competition coefficients (only for the case $c_{21}=c_{12}$ ) and three different values for $a_{12}=a_{21}$. In both panels $b_{\max }=0.2$.

varies between 3 and 10 days, that seems to be a realistic estimate (Faraj et al., 2006; Jones et al., 2012).

Figure 2 shows how the value of $\mathcal{R}_{0}$ depends on the values of the different ecological ingredients (competition coefficients $c_{12}, c_{21}$ and vector feeding preference $\delta_{V}$ ). Continuous lines represent the case when species 1 is preferred, while dashed lines when the vector prefers species 2 . On the $x$-axis $\delta_{V}$, the feeding preference index, ranges from 1 to 10. Different panels refer to different values of $\left(c_{12}, c_{21}\right)$ that assume respectively the values of $(0.1,0.5,0.9)$.

In every case we observe that $\mathcal{R}_{0}>1$, so the infection-free equilibrium will always be unstable.

The interplay of both competition and feeding preference is rather complex; however we see that they both affect significantly $\mathcal{R}_{0}$. Higher values of $\mathcal{R}_{0}$ can be observed when the vector prefers to feed on the less abundant host. For example, if species 1 is preferred and $c_{12}=0.9, c_{21}=0.1$ (i.e. species 1 is less abundant than species 2 ), we can observe that $\mathcal{R}_{0}$ reaches its maximum values. Conversely, if the most abundant species is preferred, $\mathcal{R}_{0}$ does not seem 

presented in Figure B1 in the Supplementary Material.

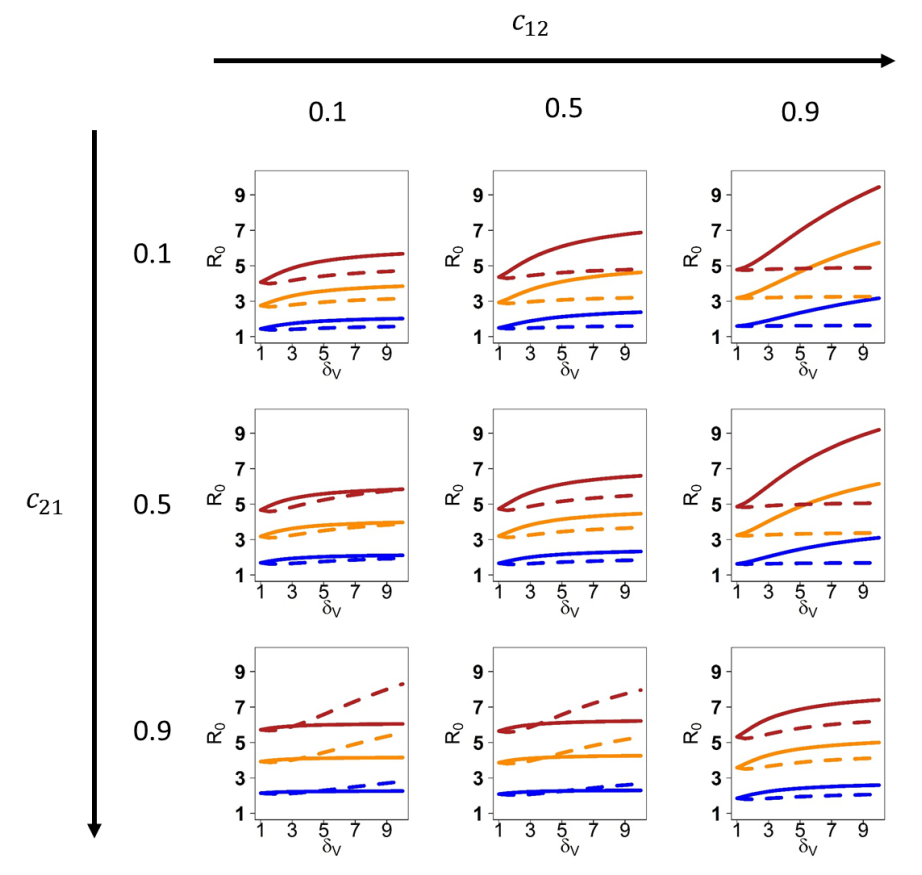

Figure 2: $\mathcal{R}_{0}$ with $b_{\max }=0.1$ (blue), 0.2 (orange) and 0.3 (red) as function of the competition coefficients $c_{12}, c_{21} \in\{0.1,0.5,0.9\} \times\{0.1,0.5,0.9\}$ and the feeding preference index $\delta_{V}$ ranging from 1 to 10 . Continuous (dashed) lines regard the case when species 1 (2) is preferred. 


\subsection{Effect of competition and shifting mosquito feeding preference on infection} seasonal dynamics

To model a typical season, we add another host type $M$, representing a mammal species, individuals of which are bitten at rate $b_{M}$. They do not have any interaction with other hosts and we assume they are a closed population. Moreover, we assume that they are dead-end hosts, so they do not infect the vector, and that they can recover and become immune for life.

Hence, we add to system (1) the three following equations

$$
\left\{\begin{array}{l}
N_{M}^{\prime}=0 \\
I_{M}^{\prime}=p_{V M} b_{M} I_{V} \frac{S_{M}}{N_{M}}-\alpha_{M} I_{M} \\
R_{M}^{\prime}=R_{M}+\alpha_{M} I_{M}
\end{array}\right.
$$

The description of the new parameters and their values are reported in Table 2. As far as we know, there are no empirical estimates for the probability of transmission to any mammal species. Hence in our simulations we consider two values for $p_{V M}$. In the first case we assume $p_{V M}=p_{V 1}=p_{V 2}=0.88$ as in (Bowman et al., 2005), in the other case we assume $p_{V M}$ value and order of magnitude less than $p_{V 1}, p_{V 2}$, i.e. $p_{V M}=0.088$.

An important ecological aspect that affects mosquito seasonal dynamics is the diapause (Denlinger and Armbuster, 2014). It is a common mechanism adopted by mosquitoes to survive winter; in the case of Culex pipiens, only adult females undergo diapause, i.e. they do not lay new eggs until the following spring. Daylight duration plays a key role in its activation (Spielman and Wong, 1973; Denlinger and Armbuster, 2014). To take into account this feature, we introduce a new variable $\gamma$, which is a function of the daylight duration as published in (Marini et al., 2016). It ranges from 0 to 1 and it is shown in Figure 3 (dotted line in panel d). The equation for $V$ in (1) is replaced by

$$
V^{\prime}=\left[n_{E} \sigma \gamma b_{\max }\left(1-\rho_{V} V\right)-\mu_{V}\right] V
$$

The simulations start on June 1 in a given year with an infected bird belonging to species 1 and lasts 6 months. $\gamma$ is modeled according to the daylight duration 
recorded at $46^{\circ} \mathrm{N}$ latitude (see Supplementary Material for more details).

Instead of simulating the deterministic system (1), we consider a Markov chain whose transition probabilities corresponds to the rates of the differential equations in (1) (see section A in the Supplementary Material for more details). We decided to follow a stochastic approach to be able to account for demographic stochasticity, relevant for instance at the invasion stage.

\begin{tabular}{llcc} 
Parameter & Description & Value & Source \\
\hline$N_{M}$ & Number of mammals & 1000 & Assumption \\
$\alpha_{M}$ & Recovery rate from WNV for dead end hosts (/day) & 0.07 & (Bowman et al., 2005) \\
$p_{V M}$ & Transmission probability mosquito to mammal & Varying &
\end{tabular}

Table 2: Mammal parameters.

a

$\delta$

the Here we present the outcome of the model when inter-species competition is
absent, i.e. $c_{12}=c_{21}=0$, the vector does not have a preferred avian species (i.e.
$\delta_{V}=1$ ) and its biting rate is $b_{\text {max }}=0.2$. So for each host type $i \in\{1,2, M\}$
the biting rate is

$$
b_{i}=b_{\max } \frac{N_{i}}{N_{1}+N_{2}+N_{M}} .
$$

Figure 3 shows the prevalence, i.e. the number of infected divided by the total number of individuals, for each host type $i$ and the vector during the season. Avian species 1 experiences a higher infection than the other two host populations. Highest prevalence in the vector is recorded very late in the season; this may be due to our assumption that towards the end of the season almost no mosquitoes reproduce, as $\gamma$ is very close to zero, and therefore the influx of susceptible vectors is very low in that period. Maximal prevalence in the host species is expected much earlier. For mammal, this occurs two months after the beginning of the season (10th of August), when $p_{V M}=p_{V 1}=p_{V 2}$ (black line in panel c), or around three months after the beginning (middle of September), 
${ }_{254}$ when $p_{V M}=p_{V 1} \cdot 10^{-1}$ (red line in panel c), similar to what happens in the avian populations.
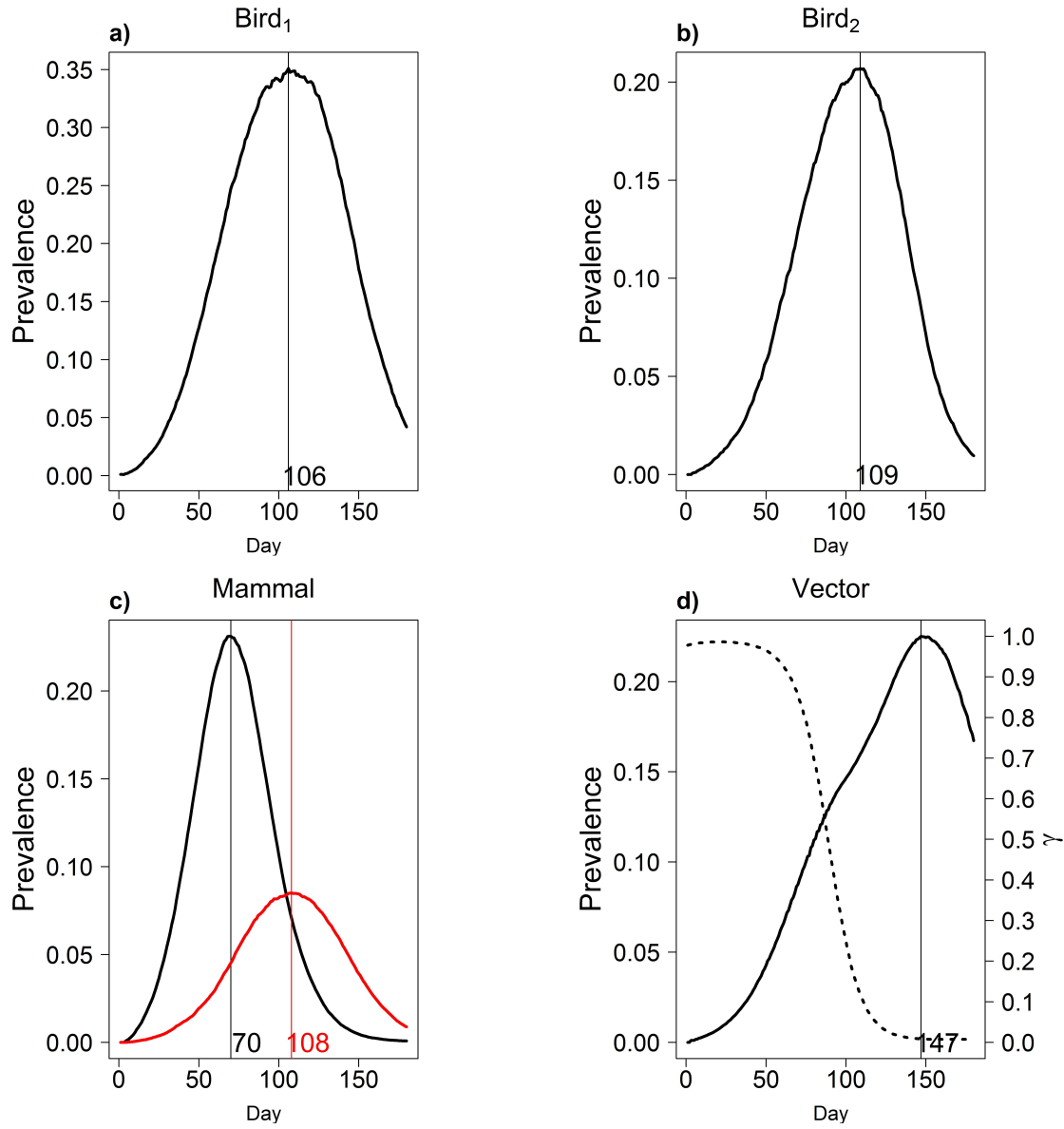

Figure 3: Prevalence for the three host types (panels a-c) and the vector (panel d). Black (red) line in panel c represent the outcome with $p_{V M}=0.88$ (0.088). The vertical lines show the day at which the maximal prevalence is reached. Parameter $\gamma$ is shown in panel $\mathrm{d}$ (dotted line). 


\subsubsection{Including feeding preference shift only}

For the sake of simplicity we assume, in this Subsection, that there is no competition, i.e. $c_{12}=c_{21}=0$, and that there is no preference between the two avian species, i.e. $\delta_{V}=1$, but there is preference between birds and mammals. In particular this preference, which shifts through the season, is modeled according to the functions presented in (Kilpatrick et al., 2006b). More specifically, we assume that at time $t$ the vector bites a host of type $i \in\{$ bird, $M\}$ with probability $f_{i}(t)$ with $f_{\text {bird }}(t)+f_{M}(t)=1$. Therefore the biting rates are

$$
b_{i}(t)=f_{i}(t) b_{\max } \quad i \in\{\operatorname{bird}, M\}
$$

with $f_{i}(t)$ as shown in Figure 4 (panels a-c) and

$$
b_{i}=b_{b i r d} \frac{N_{i}}{N_{i}+N_{j}} \quad i, j \in\{1,2\} .
$$

The inclusion of shifts in feeding preference significantly affects virus spread. As shown in Figure 4, the highest prevalences for all populations occur earlier compared to the scenario in which the biting rates are time independent (Figure 3). More precisely, they are expected about two months (beginning of August) after the introduction of the first infected bird. The two avian species exhibit a similar pattern, but we can note that, compared to the baseline case, the prevalences have a much higher maximum (about 60\%), and they decrease to zero more quickly. 

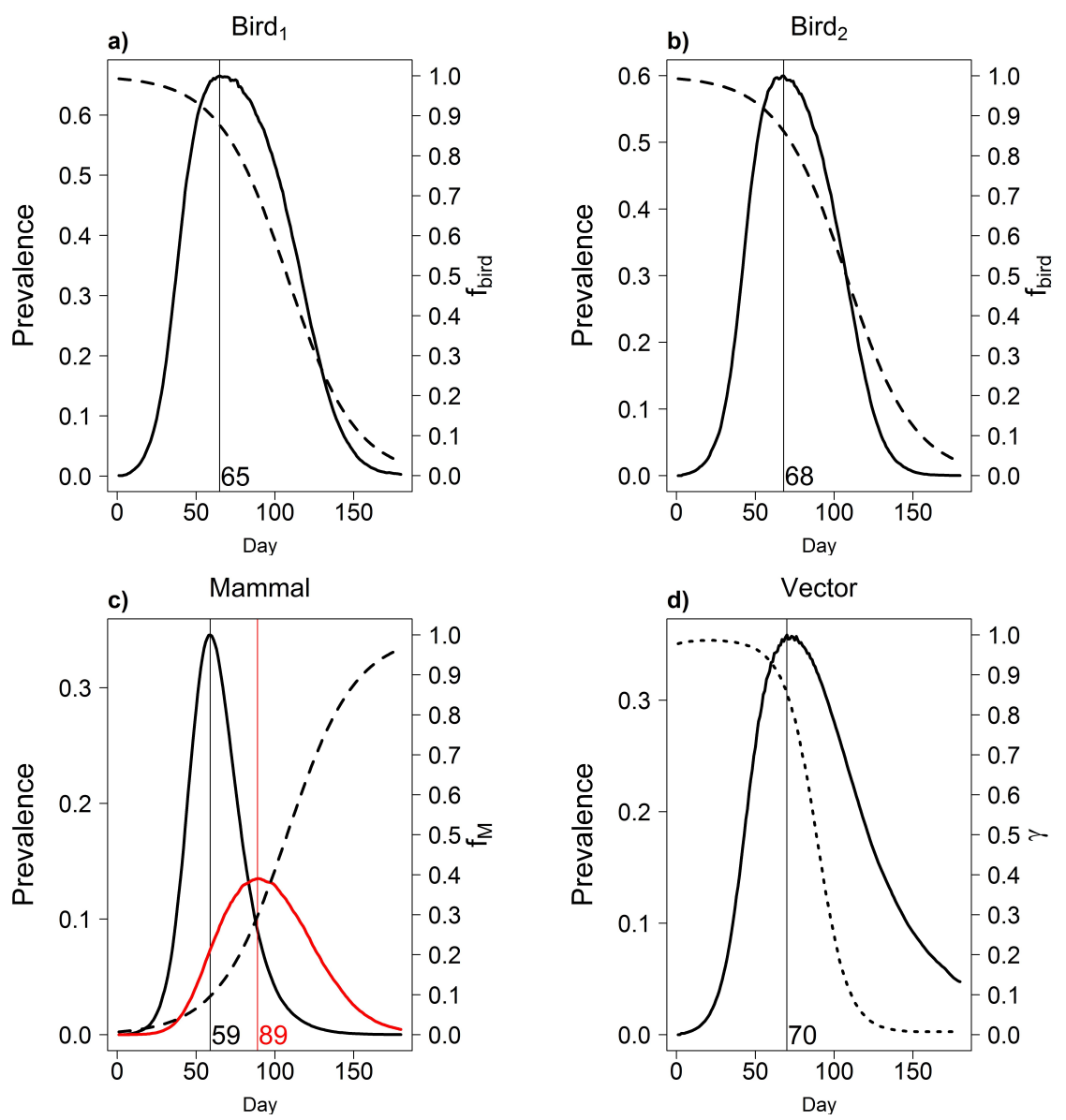

Figure 4: Prevalence for the three host types (panels a-c) and the vector (panel d). Black (red) line in panel c represent the outcome with $p_{V M}=0.88$ (0.088). The shifting mosquito feeding preferences are represented in panel a, b $\left(f_{b i r d}(t)\right)$ and $\mathrm{c}\left(f_{M}(t)\right)$ with dashed lines. The vertical lines show the times when the maximal prevalence is reached. The function $\gamma$ (diapause rate) is shown in panel d (dotted line). 


\subsubsection{The complete model without feeding preference shift}

In this case we allow $c_{12}$ and $c_{21}$ to be different from 0 , while $b_{\max }=0.2$ as previously and $p_{V M}=0.88$ as in (Bowman et al., 2005). We explore a range of $c_{12}, c_{21}$ combinations, with $\left(c_{12}, c_{21}\right) \in(0.05,1) \times(0.05,1)$. Moreover, we study three cases of vector preference for birds: no preference, preference for species 1 or for species 2 , in which cases $\delta_{V}=5$.

The inclusion of competition produces rather different outcomes, depending on vector preference, as shown in Figure 5, where the central column presents the no-preference case while the first and third column show the cases of preference for species 1 and 2 respectively. When there is no preference between the two bird species, all four populations present smaller maximal prevalences if the species with higher infectiousness (1) is less abundant, i.e. if it is severely affected by the competition with the other $\left(c_{12} \gg c_{21}\right)$. The same observation can be made for the vector in the case it prefers the more infectious bird species (1), and when this latter has a strong ecological disadvantage $\left(c_{12}>0.6\right)$. For avian species, the maximal prevalence is higher for the preferred bird population. Moreover, for the preferred avian species (say $i$ ), its highest maximal prevalence values are reached when $c_{i j} \gg c_{j i}$, i.e. when it has a strong ecological disadvantage. This corresponds to what found for the value of $\mathcal{R}_{0}$ in Section 3.1 .

If species 1 (with higher probability of infecting the vector) is preferred, then the maximal prevalence for both host and vector population is recorded much earlier in the season compared to when the vector prefers species 2 or there is no preference between them (see Figure 6 , blue boxplots). Moreover, as shown in Figure C1 in the Supplementary Material, if this host type is also extremely affected by competition with the other species, then the vector maximal prevalence is expected less than two months after the beginning of the epidemics, so much earlier with respect to cases with low competition coefficients values. 

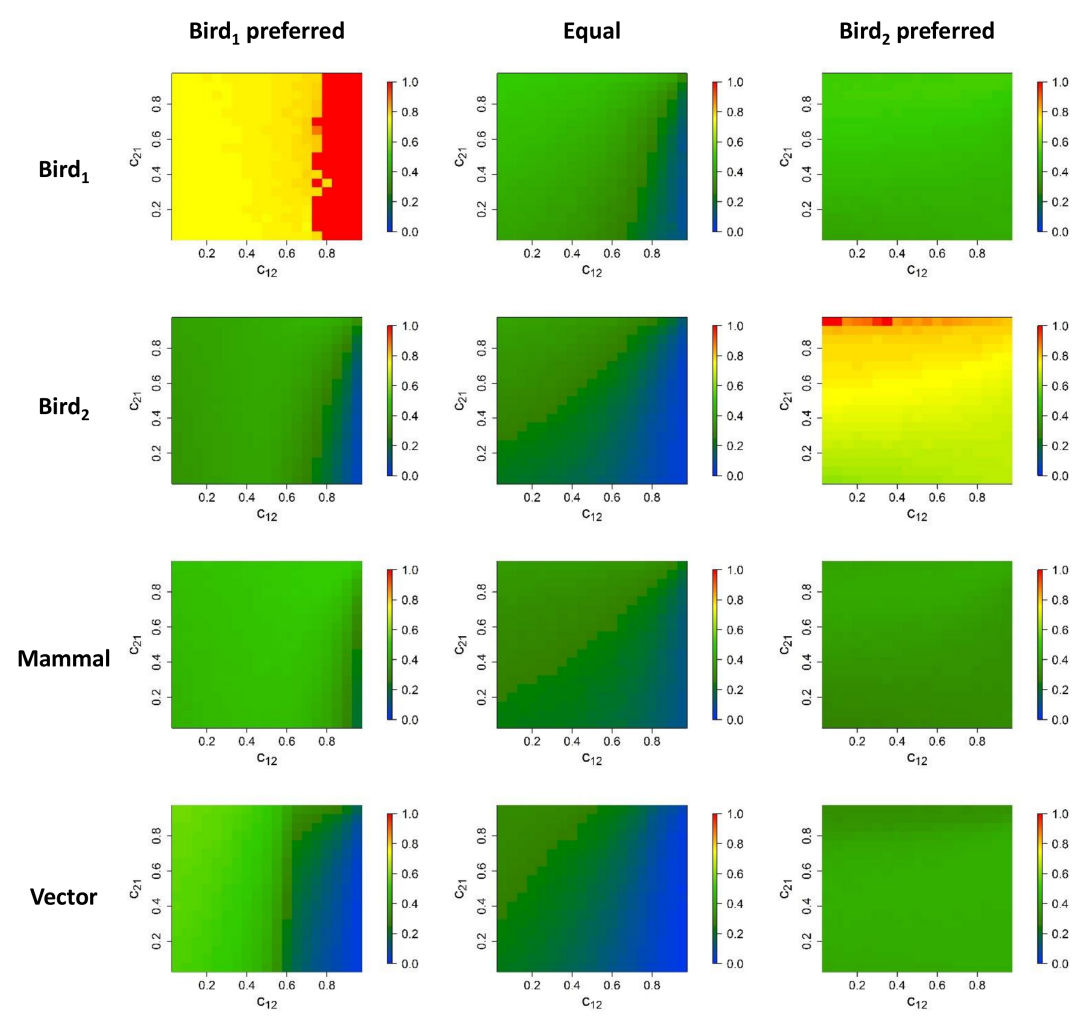

Figure 5: Maximal prevalence for the three host types and the vector for the model without feeding preference shift (Subsection 3.2.3), where species 1 (left) or species 2 (right) is preferred $\left(\delta_{V}=5\right)$, or there is no preference $\left(\delta_{V}=1\right.$, center). Values range from 0 (dark blue) to 1 (bright red). Avian species 1 is assumed to be more infectious than species $2\left(p_{1 V}>p_{2 V}\right)$. 


\subsubsection{The complete model with feeding preference shift}

In this Subsection, we study how the virus circulation is affected by competition, as in Subsection 3.2.3, but with the time-dependent biting rates as presented in Subsection 3.2.2.

As shown in Figure 7, if the vector changes its feeding preference during the season, then the expected maximal prevalence in vector, hosts and mammals increases in comparison with the case with constant preference. This is consistent with what observed in Subsection 3.2.2 where we investigated the case with time-dependent biting rates but without competition.

If there is no preference between the two bird populations (central column), it can be seen that the lowest maximal prevalences are expected when the competition is not particularly high, similarly to what we observed for $\mathcal{R}_{0}$ in Figure 1. On the other hand, if there is a preference for species $i$, its maximal prevalence is much higher than that of the other avian population. Furthermore, if the preferred host species is strongly affected by competition (large $c_{i j}$ ), both avian maximal prevalences are larger, consistent with the computation of $\mathcal{R}_{0}$ in Section 3.1 and with the simulations presented in Subsection 3.2.3, where we studied the same scenario with time-independent biting rates.

In this case, infection prevalence in mammals and vector is not significantly affected by bird competition and its value is around $50 \%$ for every $\left(c_{12}, c_{21}\right)$ combination.

As found previously in the case without preference between birds and no competition (Subsection 3.2.2), maximal prevalence occurs earlier than when

there is no shift. In fact, as shown in Figure 6, avian and vector infection prevalence peaks are expected to occur from to two to three months earlier respectively. Moreover, the maximal prevalence is recorded earlier in the season when mosquitoes prefer to feed on the more infectious avian species (see Figure 6 , orange boxplots), while there does not seem to be a significant difference between the cases $\delta_{V}=1$ and $\delta_{V}=5$ when species 2 is preferred. 
Bird $_{1}$

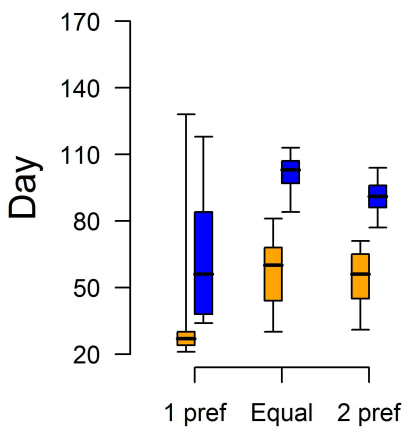

Mammal

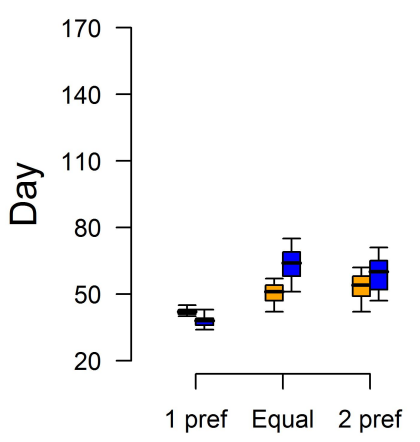

Bird $_{2}$

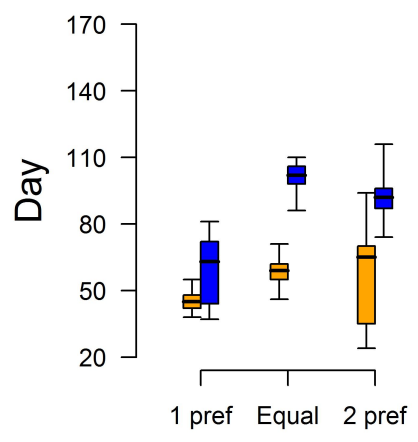

Vector

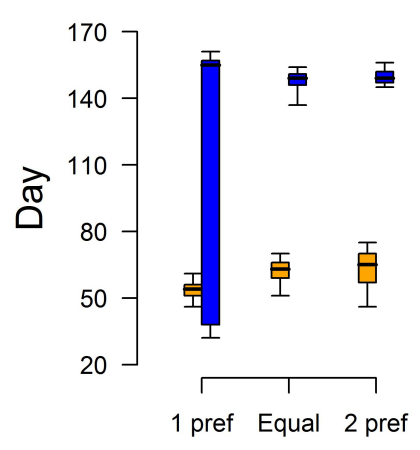

Figure 6: Boxplot of the maximal prevalence recording time (number of days after the introduction of the first infected host) in the cases with competition coefficients $\left(c_{12}, c_{21}\right) \in$ $(0.05,1) \times(0.05,1)$, and where species 1 or species 2 is preferred $\left(\delta_{V}=5\right)$, and when there is no preference $\left(\delta_{V}=1\right)$ with (without) the assumption of shifting vector feeding preference in orange (in blue). Whiskers: $2.5 \%$ and $\mathbf{9 7 . 5 \%}$ quantiles; box: $25 \%$ and $\mathbf{7 5 \%}$ quantiles; thick line: median. 

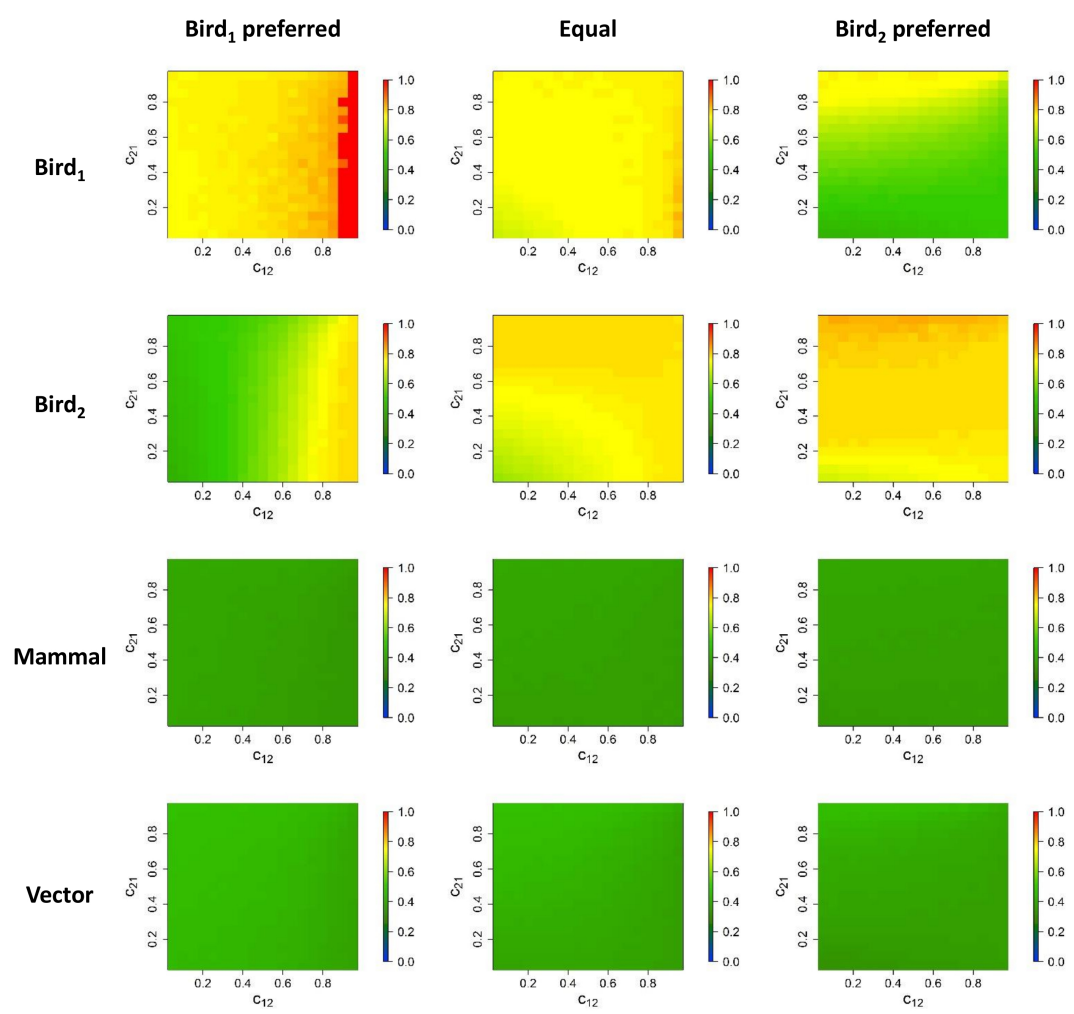

Figure 7: Maximal prevalence for the three host types and the vector in the cases with competition and time-dependent biting rates (Subsection 3.2.4), where species 1 (left) or species 2 (right) is preferred $\left(\delta_{V}=5\right)$, or there is no preference $\left(\delta_{V}=1\right.$, center). Values range from 0 (dark blue) to 1 (bright red). 


\section{Conclusions}

In this paper, we presented a mathematical framework to investigate a vector-borne infection spreading in a multi-host community where individuals can interact with each other epidemiologically as well as ecologically (in particular by competition), following the study presented in (Roberts and Heesterbeek, 2013) and the model proposed in (Lord et al., 1996).

We observed that competition may increase $\mathcal{R}_{0}$ by decreasing host population sizes (and thus increasing vector/host ratios), but that at the same time it might decrease host life expectancy and in this way decrease $\mathcal{R}_{0}$. A general pattern of the effect of competition on $\mathcal{R}_{0}$ is therefore difficult to establish, as the influence on infection dynamics very much depends on the ecological particularities of the system one studies. $\mathcal{R}_{0}$ is also strongly influenced by the vectors' biting rate, but also by vector feeding preferences, which may cause a large increase of $\mathcal{R}_{0}$ if the less abundant host is the preferred one. On the other hand, $\mathcal{R}_{0}$ might be smaller if the vector tends to feed on the less competent host.

In order to be able to obtain more precise conclusions, we focused on a particular case, the spread of West Nile Virus within an avian population composed by two different species that share the same habitat and compete for resources. We explored a wide range of values for the ecological ingredients, such as ecological interactions and vector feeding preference, using epidemiological parameter values that have been estimated for WNV. We found that $\mathcal{R}_{0}$ can be strongly influenced by competition and feeding preferences (see Figures 1 and 2).

We also used the model, parameterized for WNV, to simulate seasonal epidemics, and thus studying the effect of competition and vector preference on transient dynamics. This model included also dead-end hosts, typically mammals for WNV, and allowed for a shifting preference of vectors, from birds in the first part of the season to mammals in the second part, as shown to occur in natural systems by Kilpatrick et al. (2006b), Thiemann et al. (2011) and Burkett-Cadena et al. (2012). One effect of the presence of dead-end hosts is a dilution effect (Keesing et al., 2006), as they decrease the circulation of the 
virus by wasting, from the pathogen transmission point of view, a proportion of the vector bites. This effect is no longer observed when assuming timedependent vector feeding preference; in fact, in this case mosquitoes bite only competent hosts at the beginning of the season, enhancing the increase of infection prevalence; indeed, the virus would circulate among mosquitoes with a higher incidence than in the case when mosquitoes are assumed to feed also on mammals, which are assumed to be dead-end hosts, as studied in Subsection 3.2.1. From the simulations, it also appears that, with shifting vector preferences, infection prevalence in dead-end hosts and vectors is not influenced by bird competition (compare Figures 5 and 7 ), which in this case affects infection spread only among avian populations.

Shifting feeding preference during a season has another important consequence: the times of highest prevalence in a season are recorded around the same period for both vectors and birds, i.e. about two months after the start of the epidemics. This result agrees with actual observations. For instance, Bell et al. (2005), Lukacik et al. (2006) and Reisen et al. (2010) recorded the highest WNV prevalence in mosquitoes in August in different parts of the US, while Nemeth et al. (2007) and Kwan et al. (2010) noted that the highest records of WNV avian cases are during summer (June-July). On the other hand, if it is assumed that vector feeding preferences are fixed throughout the season (Subsections 3.2.1 and 3.2.3), one can see that the prevalence peaks later in the season and in vectors later than in birds. We argue that the assumption of changing feeding preferences is important when studying the seasonal pattern of infections in vector-borne pathogen models.

The model considered here does not attempt to be realistic for any specific infection, even though some assumptions and parameter values have been tailored for WNV. In reality, Culex mosquitoes bite a large number of bird and mammal species, some of which will be dead-end hosts, others will be of different competence for the transmission of WNV (Komar et al., 2003). The model we studied considered only two avian species, both highly competent. Possibly, the rather high prevalence of WNV in the simulations, as well as the high values of $\mathcal{R}_{0}$, are 
an artifact arising from this simplified situation. Another questionable assumption is that birds are not allowed to recover, though antibody-positive birds are not difficult to find in endemic areas (Jozan et al., 2003; Mckee et al., 2015). Including a compartment of recovered birds would not change the values of $\mathcal{R}_{0}$ but would certainly decrease infection prevalence. Despite these limitations, we believe that this study of a simplified situation gave important insights on the importance of ecological interactions and vector feeding preferences in shaping infection dynamics in a multi-host-vector system.

\section{Acknowledgements}

This work was funded by the Autonomous Province of Trento (Italy), Research funds for Grandi Progetti (Prot. n. 319292-S116-2013), Project LExEM

(Laboratory of excellence for epidemiology and modelling, http://www.lexem.eu). The funders had no role in study design, decision to publish, or preparation of the manuscript. 
Agnew, P., Haussy, C., and Michalakis, Y. Effects of density and larval competition on selected life history traits of Culex pipiens quinquefasciatus (Diptera: Culicidae). Journal of Medical Entomology, 37(5):732-755, 2010.

Bell, J., Mickelson, N., and Vaughan, J. West Nile virus in host-seeking mosquitoes within a residential neighborhood in Grand Forks, North Dakota. Vector-borne and Zoonotic Diseases, 5(4), 2005.

Bokil, V. A. and Manore, C. A. Linking population dynamics and disease models for multi-host pathogen systems: implications for pathogen and species invasion. Journal of Biological Systems, 21(4), 2013.

Bowers, R. and Turner, J. Community structure and the interplay between interspecic infection and competition. Journal of Theoretical Biology, 187: 95-109, 1997.

Bowman, C., Gumel, A., van den Driessche, P., Wu, J., and Zhu, H. A mathematical model for assessing control strategies against West Nile virus. Bullettin of Mathematical Biology, 67:1107-1133, 2005.

Burkett-Cadena, N., Hassan, H., Eubanks, M., Cupp, E., and Unnasch, T. Winter severity predicts the timing of host shifts in the mosquito Culex erraticus. Biology Letters, 8(4):567-569, 2012.

Cruz-Pacheco, G., Esteva, L., Montano-Hirose, J., and Vargas, C. Modelling the dynamics of West Nile virus. Bullettin of Mathematical Biology, 67:1157$1172,2005$.

Cruz-Pacheco, G., Esteva, L., and Vargas, C. Multi-species interactions in West Nile virus infection. Journal of Biological Dynamics, 6(2):281-298, 2012.

Denlinger, D. and Armbuster, P. Mosquito diapause. Annual Review of Entomology, 59:73-93, 2014.

Diekmann, O., Heesterbeek, J., and Roberts, M. The construction of nextgeneration matrices for compartmental epidemic models. Interface, $7(47)$ : 873-885, 2010. 
Faraj, C., Elkohli, M., and Lyagoubi, M. Cycle gonotrophique de Culex pipiens (Diptera: Culicidae), vecteur potentiel du virus West Nile, au Maroc: estimation de la dure en laboratoire. Bulletin de la Societe de pathologie exotique, 99(2):119-121, 2006.

Gamarra, J., Montoya, J., Alonso, D., and Sol, R. Competition and introduction regime shape exotic bird communities in Hawaii. Biological Invasions, 7:297$307,2005$.

Han, L. and Pugliese, A. Epidemics in two competing species. Nonlinear Analysis: Real World Applications, 10:723-744, 2009.

Hartemink, N., Davis, S., Reiter, P., Hubalek, Z., and Heesterbeek, J. The importance of bird-to-bird transmission for the establishment of West Nile virus. Vector-Borne and Zoonotic Diseases, 7:575-584, 2007.

Jones, C., Lounibos, L., Marra, P., and Kilpatrick, A. Rainfall influences survival of Culex pipiens (Diptera: Culicidae) in a residential neighborhood in the mid-Atlantic United States. Journal of Medical Entomology, 49(3):467473, 2012.

Jozan, M., Evans, R., R.McLean, Hall, R., Tangredi, B., Reed, L., and Scott, J. Detection of West Nile virus infection in birds in the United States by blocking elisa and immunohistochemistry. Vector-borne and Zoonotic Diseases, 3(3): 99-110, 2003.

Keesing, F., Holt, R., and Ostfeld, R. Effects of species diversity on disease risk. Ecology Letters, 9(4):485-498, 2006.

Kilpatrick, A. M., Daszak, P., Jones, M., Marra, P., and Kramer, L. Host heterogeneity dominates West Nile virus transmission. Proceedings of the Royal Society B, 273:2327-2333, 2006a.

Kilpatrick, A. M., Kramer, L. D., Jones, M. J., Marra, P. P., and Daszak, P. West Nile virus epidemics in North America are driven by shifts in mosquito feeding behaviour. Plos Biology, 4, 2006b. 
Komar, N., Langevin, S., Hinten, S., Nemeth, N., Edwards, E., Hettler, D., Davis, B., Bowen, R., and Bunning, M. Experimental infection of north American birds with the New York 1999 strain of West Nile virus. Emerging Infectious Diseases, 9:311-322, 2003.

Kwan, J., Kluh, S., Madon, M. B., and Reisen, W. West Nile virus emergence and persistence in Los Angeles, California, 2003-2008. American Journal of Tropical Medicine and Hygiene, 83(2):400-412, 2010.

Lord, C., Woolhousde, M., Heesterbeek, J., and Mellor, P. Vector-borne diseases and the basic reproduction number: a case study of African horse sickness. Medical and Veterinary Entomology, 10:19-28, 1996.

Lukacik, G., Anand, M., Shusas, E., Howard, J., Oliver, J., Chen, H., Backenson, P., Kauffman, E., Berndard, K., Kramer, L., and White, D. West Nile virus surveillance in mosquitoes in New York state, 2000-2004. Journal of the American Mosquito Control Association, 22(2):264-271, 2006.

Maidana, N. and Yang, H. Dynamic of West Nile virus transmission considering several coexisting avian populations. Mathematical and Computer Modelling, $53: 1247-1260,2011$.

Marini, G., Poletti, P., Giacobini, M., Pugliese, A., Merler, S., and Rosá, R. The Role of Climatic and Density Dependent Factors in Shaping Mosquito Population Dynamics: The Case of Culex pipiens in Northwestern Italy. PLOS ONE, 11(4):1-15, 042016.

Mckee, E., Walker, E., Anderson, T., Kitron, U., Brawn, J., Krebs, B., Newman, C., Ruiz, M., Levine, R., Carrington, M., McLean, R., Goldberg, T., and Hamer, G. West Nile virus antibody decay rate in free-ranging birds. Journal of Wildlife Diseases, 51(3):601-608, 2015.

Nemeth, N. M., Beckett, S., Edwards, E., Klenk, K., and Komar, N. Avian mortality surveillance for West Nile virus in Colorado. American Journal of Tropical Medicine and Hygiene, 76(3):431-437, 2007. 
Reisen, W. Ecology of West Nile Virus in North America. Viruses, 5:2079-2105, 2013.

Reisen, W., Wheeler, S., Garcia, S., and Fang, Y. Migratory birds and the dispersal of Arboviruses in California. American Journal of Tropical Medicine and Hygiene, 83(4):808-815, 2010.

Rizzoli, A., Bolzoni, L., Chadwick, E., Capelli, G., Montarsi, F., Grisenti, M., de la Puente, J. M., Munoz, J., Figuerola, J., Soriguer, R., Anfora, G., Luca, M. D., and Rosá, R. Understanding West Nile virus ecology in Europe: Culex pipiens host feeding preference in a hotspot of virus emergence. Parasites $\mathscr{G}$ Vectors, 8:213, 2015.

Roberts, M. G. and Heesterbeek, J. A. P. Characterizing the next-generation matrix and basic reproduction number in ecological epidemiology. Journal of Mathematical Biology, 66:1045-1064, 2013.

Simpson, J., Hurtado, P., Medlock, J., Molaei, G., Andreadis, T., Galvani, A., and Diuk-Wasser, M. Vector host-feeding preferences drive transmission of multi-host pathogens: West Nile virus as a model system. Proceeding of the Royal Society B-Biological Sciences, 279(1730):925-933, 2012.

Smithburn, K., Hughes, T., Burke, A., and Paul, J. A neurotropic virus isolated from the blood of a native of Uganda. American Journal of Tropical Medicine, 20:471-472, 1940 .

Spielman, A. and Wong, J. Environmental control of ovarian diapause in Culex pipiens. Annals of the Entomological Society of America, 66(4):905-907, 1973.

Taylor, L., Cummings, R., Velten, R., Collibus, K. D., Morgan, T., Nguyen, K., and Gerry, A. Host (avian) biting preference of Southern California Culex mosquitoes (Diptera: Culicidae). Journal of Medical Entomology, 49(3):687696,2012 . 
517

Thiemann, T., Wheeler, S., Barker, C., and Reisen, W. Mosquito host selection varies seasonally with host availability and mosquito density. Plos Neglected Tropical Diseases, 5(12), 2011.

Turell, M. J., Guinn, M. L. Ó., Dohm, D. J., and Jones, J. W. Vector competence of north American mosquitoes (diptera: Culicidae) for West Nile virus. Journal of Medical Entomology, 38:130-134, 2001.

Zeller, H. and Schuffenecker, I. West Nile virus: An overview of its spread in Europe and the Mediterranean basin in contrast to its spread in the Americas. European Journal of Clinical Microbiology and Infectious Diseases, 23(3):147156, 2004. 